\begin{tabular}{|c|c|c|}
\hline & $\begin{array}{c}\text { Journal of Mathematical Analysis and Modeling } \\
\text { jmam.sabapub.com } \\
\text { ISSN 2709-5924 }\end{array}$ & $\begin{array}{c}\text { J Math Anal \& Model } \\
\text { (2020)1(1) : 33-46 } \\
\text { doi:10.48185/jmam.v1i1.2 }\end{array}$ \\
\hline & & \\
\hline
\end{tabular}

\title{
Further results on the existence of solutions for generalized fractional quadratic functional integral equations
}

\author{
Mohammed S. ABdo* \\ Department of Mathematics, Hodeidah University, Al-Hodeidah, Yemen.
}

\begin{abstract}
- Received: 01.10.2020 • Accepted/Published Online: 21.11.2020 • Final Version: 03.12.2020
Abstract

This paper discusses some existence results for at least one continuous solution for generalized fractional quadratic functional integral equations. Some results on nonlinear functional analysis including Schauder fixed point theorem are applied to establish the existence result for proposed equations. We improve and extend the literature by incorporated of some well known and commonly cited results as special cases in this topic. Further, we prove the existence of maximal and minimal solutions for these equations.
\end{abstract}

Keywords: Fractional differential equations, $\psi$-Caputo fractional derivative, Existence, Maximal and minimal solutions, Fixed point theorem.

2010 MSC: 34A08, 26A33, 49J35.

\section{Introduction}

In the past few years, many author have utilized the fractional calculus as a path of describing natural phenomena in diverse fields such as mathematics, applied science, and engineering. Recently, this topic has aroused deep intense interest, and increasing studies of some researchers, both in mathematics and in applications. As a result of this interest, many studies have been presented on this topic. In this regard, we refer to the monographs of leading researchers $[1,2,3,4,5,6,7]$, and the papers $[8,9,10,11,12]$. There are various definitions of fractional derivatives and fractional integrals, the most famous of which are Riemann-Liouville [4], Caputo [13], Hilfer [2], Hadamard [14], Katugampola [15] and Prabhakar [16]. On the other hand, new, more general, local, and non-local fractional operators have emerged incorporates several operators dealing with a different kernel of integrations, such as Caputo-Hadamard [17], Caputo-Katugampola [18], Caputo-Fabrizio [19], Atangana-Baleanu-Caputo [20], $\psi$-Caputo [21], $\psi$-Hilfer [22] and some other operators $[23,24,25]$. These operators combine a wide class of fractional derivatives as the aforementioned ones. It is notable that integral equations (IEs) have many useful applications in describing various events and problems of the real world. Additionally, the theory of IEs is quickly developing utilizing the instruments of fixed point

${ }^{*}$ Corresponding author: msabdo1977@gmail.com

(c) 2020 SABA. All Rights Reserved. 
theory and nonlinear analysis. Specifically, quadratic integral equations (QIEs) have numerous helpful applications in the real world. For instance, QIEs are regularly pertinent in the theory of radiative exchange, the theory of neutron transport, the dynamic theory of gases, traffic theory, and queuing theory. Numerous creators studied the existence of solutions for sundry classes of nonlinear QIEs (see e.g. [26, 27, 28, 29, 30, 31, 32, 33, 34] and $[35,36,37,38,39,40]$. Notwithstanding, in a large portion of the above literature, the main results were investigated with the assistance of the method associated with the measure of noncompactness and the classical fractional operators. Rather than utilizing the method of measure of noncompactness, we use Schauder fixed point theorem. Moreover, our results will be more generalized of the above literature due to the kernel of integration depends on another function $\psi$, thus our obtained results will cover a large number of results for different functions of $\psi$.

In this regard, we discuss the existence of at least one continuous solution of the nonlinear fractional quadratic functional integral equation

$$
\omega(t)=h(t)+g\left(t, \omega(\varphi(t)) \int_{0}^{t} \frac{[\psi(t)-\psi(\rho)]^{\alpha-1}}{\Gamma(\alpha)} \psi^{\prime}(\rho) f(\rho, \omega(\phi(\rho)) d \rho, t \in \Delta=[0,1],\right.
$$

The equation (1.1) we can express it as

$$
\omega(t)=h(t)+g\left(t, \omega(\varphi(t)) \mathcal{J}_{0^{+}}^{\alpha, \psi} f(t, \omega(\phi(t)), t \in \Delta=[0,1],\right.
$$

where $\alpha>0, \psi: \Delta \rightarrow \mathbb{R}$ that is an increasing differentiable function such that $\psi^{\prime}(\mathrm{t}) \neq 0$ for all $\mathrm{t} \in \Delta, \mathcal{J}_{0^{+}}^{\alpha, \psi}$ is generalized fractional operator in Reimann-Liouville sense, and $h, g, \varphi, f, \phi$ are given functions satisfying some suppositions that will be specified in the next section. Moreover, the existence of maximal and minima solutions of (1.1) will be proved. For some recent results involving $\psi$-fractional derivatives, one can refer to [41, $42,43,44,45]$.

In the next analysis, we shall use the following as an abbreviation:

Fractional Quadratic Functional Integral Equation (FQFIE); Fractional Quadratic Integral Equation (FQIE); Fractional Functional Integral Equation (FFIE); Fractional Functional Differential Equation (FFDE); Fractional Functional Differential Equations (FDEs); Differential Equations (DEs); Reimann-Liouville (RL).

Remark 1.1.

1. If $\psi(t)=t$, then FQFIE (1.1) reduces to the following equation

$$
\omega(t)=h(t)+g\left(t, \omega(\varphi(t)) \int_{0}^{t} \frac{(t-\rho)^{\alpha-1}}{\Gamma(\alpha)} f(\rho, \omega(\phi(\rho)) d \rho, t \in \Delta, \alpha>0\right.
$$

which have been studied by EL-Sayed and Hashem in [46].

2. If $\psi(t)=\varphi(t)=\phi(t)=t$ then the existence of continuous solutions of

$$
\omega(t)=h(t)+g\left(t, \omega(t) \int_{0}^{t} \frac{(t-\rho)^{\alpha-1}}{\Gamma(\alpha)} f(\rho, \omega(\rho) d \rho, t \in \Delta, \alpha>0\right.
$$

was proved in [34, 47]. 
3. If $\psi(t)=\phi(t)=t$ and $g(t, \omega(\varphi(t)) \equiv 1$, then the existence of continuous solutions of

$$
\omega(t)=h(t)+\int_{0}^{t} \frac{(t-\rho)^{\alpha-1}}{\Gamma(\alpha)} f(\rho, \omega(\rho) d \rho, t \in \Delta, \alpha>0
$$

was proved in [40].

4. If $\psi(t)=\phi(t)=t, \alpha=1, g\left(t, \omega(\varphi(t)) \equiv 1\right.$, and $h(t)=\omega_{0}$, then the existence of continuous solutions of

$$
\omega(t)=\omega_{0}+\int_{0}^{t} f(\rho, \omega(\rho)) d \rho, t \in \Delta,
$$

was proved in [48].

To our knowledge, no contributes to study of the fractional quadratic functional integral equations exist in the literature, especially for those involving the $\psi$-fractional derivatives.

This paper is structured as follows: In Section 2, we recall some fundamental definitions and fact preliminary. Section 3 contains the existence result obtained by using Schauder fixed point theorem. Applications are given in Section 4. We discuss the maximal and minimal solutions of (1.1) in Section 5.

\section{Preliminaries}

Let $\mathbb{R}$ be the set of real numbers whereas $\Delta=[0,1] \subset \mathbb{R}$. $C(\Delta)$ be the Banach space of all real continuous functions defined on $\Delta$ with the supremum norm $\|\cdot\|_{\infty}$, and let $\mathrm{L}^{1}(\Delta)$ be the space of Lebesgue integrable functions on $\Delta$. The existence results will be rely on the following definitions, lemmas and theorems.

Definition 2.1. [4] The generalized RL fractional integral and derivative of order $\alpha>0$ for an integrable function $z:[a, b] \rightarrow \mathbb{R}$ with respect to another function $\psi$ are defined as follows

$$
\mathcal{J}_{a^{+}}^{\alpha, \psi} z(t)=\frac{1}{\Gamma(\alpha)} \int_{a}^{t} \psi^{\prime}(\rho)(\psi(t)-\psi(\rho))^{\alpha-1} z(\rho) d \rho, t>a,
$$

and

$$
R^{2} \mathcal{D}_{a^{+}}^{\alpha, \psi} Z(t)=\mathcal{D}^{n, \psi} \mathcal{J}_{a^{+}}^{n-\alpha, \psi} Z(t), t>a,
$$

respectively, where $\psi:[a, b] \rightarrow \mathbb{R}$ is an increasing differentiable function such that $\psi^{\prime}(t) \neq$ 0 , for all $\mathrm{t} \in[\mathrm{a}, \mathrm{b}], \mathcal{D}^{\mathrm{n}, \psi}=\left(\frac{1}{\psi^{\prime}(\mathrm{t})} \frac{\mathrm{d}}{\mathrm{dt}}\right)^{\mathrm{n}}, \mathrm{n}=[\alpha]+1$, and $\Gamma(\cdot)$ the Euler gamma function is defined by

$$
\Gamma(\delta)=\int_{0}^{\infty} \mathrm{t}^{\delta-1} \mathrm{e}^{-\mathrm{t}} \mathrm{dt}, \quad \delta \in \mathbb{C}, \mathcal{R}(\delta)>0 .
$$

Definition 2.2. [21] The generalized Caputo fractional derivative of order $\alpha>0$ with respect to another function $\psi$ can be represented by the expression

$$
C_{\mathcal{D}_{a^{+}}^{\alpha, \psi}} \mathcal{L}(\mathrm{t})=\mathcal{J}_{\mathrm{a}^{+}}^{\mathrm{n}-\alpha ; \psi} \mathcal{D}^{\mathrm{n}, \psi} z(\mathrm{t}),
$$


where $z, \psi \in \mathrm{C}^{\mathfrak{n}}[\mathrm{a}, \mathrm{b}]$. Given $z \in \mathrm{C}^{\mathfrak{n}-1}[\mathrm{a}, \mathrm{b}]$, we have

$$
\mathrm{C}_{\mathcal{D}_{a^{+}}^{\alpha, \psi}} z(\mathrm{t})={ }^{R L} \mathcal{D}_{a^{+}}^{\alpha, \psi}\left[z(t)-\sum_{j=0}^{n-1} \frac{z_{\psi}^{[j]}(a)}{j !}(\psi(t)-\psi(a))^{j}\right],
$$

where $z_{\psi}^{[j]}(t)=\left[\frac{1}{\psi^{\prime}(t)} \frac{d}{d t}\right]^{j} z(t)$, and $n=[\alpha]+1$ for $\alpha \notin \mathbb{N}$. Further, if $\alpha=n \in \mathbb{N}$, then $\mathrm{C}_{\mathcal{D}_{\mathrm{a}^{+}}^{\alpha, \psi}}^{\alpha(\mathrm{t})}=z_{\psi}^{[\mathrm{n}]}(\mathrm{t})$.

In particular, if $0<\alpha<1$, then ${ }^{C} \mathcal{D}_{a^{+}}^{\alpha, \psi} z(t)={ }^{R L} \mathcal{D}_{a^{+}}^{\alpha, \psi}[z(t)-Z(a)]$.

Remark 2.3. In Defintions 2.1 and 2.2, if $\psi(t)=t$, then equations (2.1), (2.2) and (2.3) reduce to the following equations

$$
\begin{gathered}
\left.\mathcal{J}_{a^{+}}^{\alpha} \mathcal{Z}(t)=\frac{1}{\Gamma(\alpha)} \int_{a}^{t}(t-\rho)\right)^{\alpha-1} \mathcal{Z}(\rho) d \rho, t>a, \\
R L \mathcal{D}_{a^{+}}^{\alpha} \mathcal{Z}(t)=\mathcal{D}^{n} \mathcal{J}_{a^{+}}^{n-\alpha} \mathcal{Z}(t), t>a,
\end{gathered}
$$

and

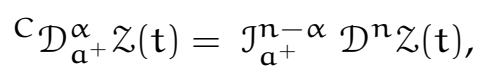

respectivaly, where $\mathcal{D}^{n}=\left(\frac{d}{d t}\right)^{n}$ and $n=[\alpha]+1$. For more details see [4].

Lemma 2.4. [21] Let $\alpha>0, \psi, z \in \mathrm{C}^{n-1}[\mathrm{a}, \mathrm{b}]$ and $z^{(\mathrm{n})}$ exists a.e. on any bounded interval of $[a, b]$. Then

$$
\mathcal{J}_{a^{+}}^{\alpha, \psi} C_{\mathcal{D}_{a^{+}}^{\alpha, \psi}}^{\alpha(t)}=z(t)-\sum_{j=0}^{n-1} \frac{z_{\psi}^{[j]}(a)}{j !}(\psi(t)-\psi(a))^{j} .
$$

In particular, if $0<\alpha<1$, we have $\mathcal{J}_{\mathrm{a}^{+}}^{\alpha, \psi} \mathrm{C}_{\mathcal{D}_{\mathrm{a}^{+}}^{\alpha, \psi}} \mathrm{Z}(\mathrm{t})=\mathrm{Z}(\mathrm{t})-\mathrm{Z}(\mathrm{a})$.

Lemma 2.5. [4] Let $\alpha>0$ and $\beta>0$. Then, we have

$$
\begin{gathered}
\mathcal{J}_{\mathbf{a}^{+}}^{\alpha, \psi} \mathcal{J}_{\mathbf{a}^{+}}^{\beta, \psi} Z(t)=\mathcal{J}_{\mathbf{a}^{+}}^{\alpha+\beta, \psi} \mathcal{Z}(\mathrm{t}), \mathrm{t} \in[\mathrm{a}, \mathrm{b}], \text { and } \\
\mathcal{J}_{\mathbf{a}^{+}}^{\alpha ; \psi}(\psi(t)-\psi(\mathrm{a}))^{\beta-1}=\frac{\Gamma(\beta)}{\Gamma(\beta+\alpha)}(\psi(t)-\psi(a))^{\beta+\alpha-1} .
\end{gathered}
$$

Lemma 2.6. $[41,21]$ Let $\alpha>0, z \in C[a, b]$ and $\psi \in \mathrm{C}^{1}[\mathrm{a}, \mathrm{b}]$. Then $\mathcal{J}_{\mathrm{a}^{+}}^{\alpha ; \psi} z \in \mathrm{C}[\mathrm{a}, \mathrm{b}]$ and

$$
\mathcal{J}_{\mathbf{a}^{+}}^{\alpha ; \psi} Z(a)=\lim _{t \rightarrow a^{+}} \mathcal{J}_{\mathbf{a}^{+}}^{\alpha ; \psi} Z(t)=0 .
$$

Theorem 2.7. (Schauder Fixed Point Theorem [49]) Let $₫$ a Banach space and $\mathrm{P}$ be a nonempty, convex, compact subset of $\varpi$. If $\mathcal{N}: \mathrm{P} \rightarrow \mathrm{P}$ is a continuous mapping, then $\mathcal{N}$ has at least one fixed point in $\mathrm{P}$. 


\section{Existence of Continuous Solutions}

Now, FQFIE (1.1) will be investigated under the following hypotheses:

(i) $h: \Delta \rightarrow \mathbb{R}$ is continuous and bound with $\theta_{1}=\sup _{t \in \Delta}|h(t)|$.

(ii) $g: \Delta \times \mathbb{R} \rightarrow \mathbb{R}$ is continuous and bounded with $\theta_{2}=\sup _{(t, \omega) \in \Delta \times \mathbb{R}}|g(t, \omega)|$.

(iii) There exist $\ell_{1}, \ell_{2}>0$ such that

$$
|g(t, \omega)-g(\rho, \varpi)| \leqslant \ell_{1}|t-\rho|+\ell_{2}|\omega-\varpi|,
$$

for all $t, \rho \in \Delta$ and $\omega, \varpi \in \mathbb{R}$.

(iv) $f: \Delta \times \mathbb{R} \rightarrow \mathbb{R}$ is satisfies Carathéodory conditions, i.e.

$f(\cdot, \omega)$ is measurable for each fixed $\omega \in \mathbb{R}$ and $f(t, \cdot)$ continuous for each fixed $t \in \Delta$.

(v) There exist constant $b$ and function $\eta \in L^{1}(\Delta)$ such that $|f(t, \omega)| \leqslant \eta(t)+b|\omega|$, for each $t \in \Delta, \omega \in \mathbb{R}$, and $\theta_{3}=\sup _{t \in \Delta} \mathcal{J}_{0^{+}}^{\beta, \psi} \eta(t)$, for any $\beta \leqslant \alpha$.

(vi) $\varphi, \phi: \Delta \rightarrow \Delta$ are continuous.

(vii) The inequality $\theta_{2} b[\psi(1)-\psi(0)]^{\alpha}<\Gamma(\alpha+1)$ is satisfied.

Theorem 3.1. Assume that (i)-(vii) be satisfied. Then FQFIE (1.1) has at least one solution $\omega(t)$ in the space $C(\Delta)$.

Proof. Fix a number $\lambda>0$, we consider the ball

$$
\mathbb{P}_{\lambda}=\{\omega \in C(\Delta):|\omega(t)| \leqslant \lambda \text { for } \mathrm{t} \in \Delta\} \subset \mathrm{C}(\Delta),
$$

with

$$
\lambda \geqslant \frac{\theta_{1}+\frac{\theta_{2} \theta_{3}}{\Gamma(\alpha-\beta+1)}[\psi(1)-\psi(0)]^{\alpha-\beta}}{1-\frac{\theta_{2} b}{\Gamma(\alpha+1)}[\psi(1)-\psi(0)]^{\alpha}} .
$$

Let the operator $\mathcal{N}: \mathrm{C}(\Delta) \rightarrow \mathrm{C}(\Delta)$ defined on $\mathbb{P}_{\lambda}$ by

$$
(\mathcal{N} \omega)(t)=h(t)+g\left(t, \omega(\varphi(t)) \int_{0}^{t} \frac{[\psi(t)-\psi(\rho)]^{\alpha-1}}{\Gamma(\alpha)} \psi^{\prime}(\rho) f(\rho, \omega(\phi(\rho)) d \rho, t \in \Delta .\right.
$$


Then, in light of our hypotheses, for $t \in \Delta$ and $\omega \in \mathbb{P}_{\lambda}$, we have

$$
\begin{aligned}
|(\mathcal{N} \omega)(t)| \leqslant & |h(t)|+\mid g\left(t, \omega(\varphi(t))\left|\int_{0}^{t} \frac{[\psi(t)-\psi(\rho)]^{\alpha-1}}{\Gamma(\alpha)} \psi^{\prime}(\rho)\right| f(\rho, \omega(\phi(\rho)) \mid d \rho\right. \\
\leqslant & \theta_{1}+\theta_{2} \int_{0}^{t} \frac{[\psi(t)-\psi(\rho)]^{\alpha-1}}{\Gamma(\alpha)} \psi^{\prime}(\rho)[\eta(\rho)+b|\omega(\phi(\rho))|] d \rho \\
= & \theta_{1}+\theta_{2} J_{0^{+}}^{\alpha-\beta, \psi}\left(\mathcal{J}_{0^{+}}^{\beta, \psi} \eta(t)\right) \\
& +\theta_{2} b \int_{0}^{t} \frac{[\psi(t)-\psi(\rho)]^{\alpha-1}}{\Gamma(\alpha)} \psi^{\prime}(\rho)|\omega(\phi(\rho))| d \rho \\
\leqslant & \theta_{1}+\theta_{2} \theta_{3} \int_{0}^{t} \frac{[\psi(t)-\psi(\rho)]^{\alpha-\beta-1}}{\Gamma(\alpha-\beta)} \psi^{\prime}(\rho) d \rho \\
& +\theta_{2} b \lambda \int_{0}^{t} \frac{[\psi(t)-\psi(\rho)]^{\alpha-1}}{\Gamma(\alpha)} \psi^{\prime}(\rho) d \rho \\
\leqslant & \theta_{1}+\frac{\theta_{2} \theta_{3}}{\Gamma(\alpha-\beta+1)}[\psi(1)-\psi(0)]^{\alpha-\beta}+\frac{\theta_{2} b \lambda}{\Gamma(\alpha+1)}[\psi(1)-\psi(0)]^{\alpha} .
\end{aligned}
$$

By assumption (vii) and inequality (3.1) we conclude that $\mathcal{N}: \mathbb{P}_{\lambda} \rightarrow \mathbb{P}_{\lambda}$.

Now, we prove that $\left\{\mathcal{N} \omega: \omega \in \mathbb{P}_{\lambda}\right\}$ is equi-continuous. For $t_{1}, t_{2} \in \Delta\left(t_{1}<t_{2}\right)$, we have

$$
\begin{aligned}
& (\mathcal{N} \omega)\left(t_{2}\right)-(\mathcal{N} \omega)\left(t_{1}\right) \\
= & h\left(t_{2}\right)-h\left(t_{1}\right)+g\left(t_{2}, \omega\left(\varphi\left(t_{2}\right)\right) \mathcal{J}_{0^{+}}^{\alpha, \psi} f\left(t_{2}, \omega\left(\phi\left(t_{2}\right)\right)\right.\right. \\
& -g\left(t_{1}, \omega\left(\varphi\left(t_{1}\right)\right) \mathcal{J}_{0^{+}}^{\alpha, \psi} f\left(t_{1}, \omega\left(\phi\left(t_{1}\right)\right)\right.\right. \\
& +g\left(t_{1}, \omega\left(\varphi\left(t_{1}\right)\right) \mathcal{J}_{0^{+}}^{\alpha, \psi} f\left(t_{2}, \omega\left(\phi\left(t_{2}\right)\right)\right.\right. \\
& -g\left(t_{1}, \omega\left(\varphi\left(t_{1}\right)\right) \mathcal{J}_{0^{+}}^{\alpha, \psi} f\left(t_{2}, \omega\left(\phi\left(t_{2}\right)\right)\right.\right. \\
= & h\left(t_{2}\right)-h\left(t_{1}\right)+\left(g \left(t_{2}, \omega\left(\varphi\left(t_{2}\right)\right)\right.\right. \\
& -g\left(t_{1}, \omega\left(\varphi\left(t_{1}\right)\right)\right) \mathcal{J}_{0^{+}}^{\alpha, \psi} f\left(t_{2}, \omega\left(\phi\left(t_{2}\right)\right)\right. \\
& +\left(g ( t _ { 1 } , \omega ( \varphi ( t _ { 1 } ) ) ) \left(\mathcal { J } _ { 0 ^ { + } } ^ { \alpha , \psi } f \left(t_{2}, \omega\left(\phi\left(t_{2}\right)\right)-\mathcal{J}_{0^{+}}^{\alpha, \psi} f\left(t_{1}, \omega\left(\phi\left(t_{1}\right)\right),\right.\right.\right.\right.
\end{aligned}
$$

but

$$
\begin{aligned}
& \mathcal{J}_{0^{+}}^{\alpha, \psi} f\left(t_{2}, \omega\left(\phi\left(t_{2}\right)\right)-\mathcal{J}_{0^{+}}^{\alpha, \psi} f\left(t_{1}, \omega\left(\phi\left(t_{1}\right)\right)\right.\right. \\
= & \int_{0}^{t_{2}} \frac{\left[\psi\left(t_{2}\right)-\psi(\rho)\right]^{\alpha-1}}{\Gamma(\alpha)} \psi^{\prime}(\rho) f\left(\rho, \omega(\phi(\rho)) d \rho+\int_{t_{1}}^{t_{2}} \frac{\left[\psi\left(t_{2}\right)-\psi(\rho)\right]^{\alpha-1}}{\Gamma(\alpha)}\right. \\
& \times \psi^{\prime}(\rho) f\left(\rho, \omega(\phi(\rho)) d \rho-\int_{0}^{t_{1}} \frac{\left[\psi\left(t_{1}\right)-\psi(\rho)\right]^{\alpha-1}}{\Gamma(\alpha)} \psi^{\prime}(\rho) f(\rho, \omega(\phi(\rho)) d \rho\right. \\
\leqslant & \int_{0}^{t_{1}} \frac{\left[\psi\left(t_{1}\right)-\psi(\rho)\right]^{\alpha-1}}{\Gamma(\alpha)} \psi^{\prime}(\rho) f\left(\rho, \omega(\phi(\rho)) d \rho+\int_{t_{1}}^{t_{2}} \frac{\left[\psi\left(t_{2}\right)-\psi(\rho)\right]^{\alpha-1}}{\Gamma(\alpha)}\right. \\
& \times \psi^{\prime}(\rho) f\left(\rho, \omega(\phi(\rho)) d \rho-\int_{0}^{t_{1}} \frac{\left[\psi\left(t_{1}\right)-\psi(\rho)\right]^{\alpha-1}}{\Gamma(\alpha)} \psi^{\prime}(\rho) f(\rho, \omega(\phi(\rho)) d \rho\right. \\
= & \int_{t_{1}}^{t_{2}} \frac{\left[\psi\left(t_{2}\right)-\psi(\rho)\right]^{\alpha-1}}{\Gamma(\alpha)} \psi^{\prime}(\rho) f\left(\rho, \omega(\phi(\rho)) d \rho=J_{t_{1}^{+}}^{\alpha, \psi} f\left(t_{2}, \omega\left(\phi\left(t_{2}\right)\right) .\right.\right.
\end{aligned}
$$


Then

$$
\begin{aligned}
& \mid \mathcal{J}_{0^{+}}^{\alpha, \psi} f\left(t_{2}, \omega\left(\phi\left(t_{2}\right)\right)-\mathcal{J}_{0^{+}}^{\alpha, \psi} f\left(t_{1}, \omega\left(\phi\left(t_{1}\right)\right) \mid\right.\right. \\
\leqslant & \mathcal{J}_{t_{1}^{+}}^{\alpha, \psi} \mid f\left(t_{2}, \omega\left(\phi\left(t_{2}\right)\right)\left|\leqslant \mathcal{J}_{t_{1}^{+}}^{\alpha, \psi} \eta\left(t_{2}\right)+b \mathcal{J}_{t_{1}^{+}}^{\alpha, \psi}\right| \omega\left(\phi\left(t_{2}\right) \mid\right.\right. \\
\leqslant & \mathcal{J}_{t_{1}^{\alpha}}^{\alpha-\beta, \psi} \mathcal{J}_{t_{1}}^{\beta, \psi} \eta\left(t_{2}\right)+b \mathcal{J}_{t_{1}^{+}}^{\alpha, \psi} \mid \omega\left(\phi\left(t_{2}\right) \mid\right. \\
\leqslant & \theta_{3} \frac{\left[\psi\left(t_{2}\right)-\psi\left(t_{1}\right)\right]^{\alpha-\beta}}{\Gamma(\alpha-\beta+1)}+b \lambda \frac{\left[\psi\left(t_{2}\right)-\psi\left(t_{1}\right)\right]^{\alpha}}{\Gamma(\alpha+1)} .
\end{aligned}
$$

Hence, the inequality (3.2) becomes

$$
\begin{array}{cl} 
& \left|(\mathcal{N} \omega)\left(t_{2}\right)-(\mathcal{N} \omega)\left(t_{1}\right)\right| \\
\leqslant \quad & \left|h\left(t_{2}\right)-h\left(t_{1}\right)\right|+\left(\ell_{1}\left|t_{2}-t_{1}\right|+\ell_{2} \mid \omega\left(\varphi\left(t_{2}\right)-\omega\left(\varphi\left(t_{1}\right) \mid\right) J_{0^{+}}^{\alpha, \psi} \mid f\left(t_{2}, \omega\left(\phi\left(t_{2}\right)\right) \mid\right.\right.\right. \\
& +\left(g\left(t_{1}, \omega\left(\varphi\left(t_{1}\right)\right)\right)\left(\theta_{3} \frac{\left[\psi\left(t_{2}\right)-\psi\left(t_{1}\right)\right]^{\alpha-\beta}}{\Gamma(\alpha-\beta+1)}+b \lambda \frac{\left[\psi\left(t_{2}\right)-\psi\left(t_{1}\right)\right]^{\alpha}}{\Gamma(\alpha+1)}\right)\right. \\
\leqslant \quad & \left|h\left(t_{2}\right)-h\left(t_{1}\right)\right|+\left(\ell_{1}\left|t_{2}-t_{1}\right|+\ell_{2}\left|\omega\left(t_{2}\right)-\omega\left(t_{1}\right)\right|\right) \mathcal{J}_{0^{+}}^{\alpha, \psi}\left(\eta\left(t_{2}\right)+b \mid \omega\left(\phi\left(t_{2}\right) \mid\right)\right. \\
& +\theta_{2} \theta_{3} \frac{\left[\psi\left(t_{2}\right)-\psi\left(t_{1}\right)\right]^{\alpha-\beta}}{\Gamma(\alpha-\beta+1)}+\theta_{2} b \lambda \frac{\left[\psi\left(t_{2}\right)-\psi\left(t_{1}\right)\right]^{\alpha}}{\Gamma(\alpha+1)} \\
\leqslant \quad & \left|h\left(t_{2}\right)-h\left(t_{1}\right)\right|+\theta_{3} \frac{\left[\psi\left(t_{2}\right)-\psi(0)\right]^{\alpha-\beta}}{\Gamma(\alpha-\beta+1)}\left(\ell_{1}\left|t_{2}-t_{1}\right|+\ell_{2}\left|\omega\left(t_{2}\right)-\omega\left(t_{1}\right)\right|\right) \\
& +b \lambda \frac{\left[\psi\left(t_{2}\right)-\psi(0)\right]^{\alpha}}{\Gamma(\alpha+1)}\left(\ell_{1}\left|t_{2}-t_{1}\right|+\ell_{2}\left|\omega\left(t_{2}\right)-\omega\left(t_{1}\right)\right|\right) \\
+ & \theta_{2} \theta_{3} \frac{\left[\psi\left(t_{2}\right)-\psi\left(t_{1}\right)\right]^{\alpha-\beta}}{\Gamma(\alpha-\beta+1)}+\theta_{2} b \lambda \frac{\left[\psi\left(t_{2}\right)-\psi\left(t_{1}\right)\right]^{\alpha}}{\Gamma(\alpha+1)} \\
\rightarrow \quad 0 \text { as } t_{2} \rightarrow t_{1} .
\end{array}
$$

This shows that $\left\{\mathcal{N} \omega: \omega \in \mathbb{P}_{\lambda}\right\}$ is equi-continuous on $\Delta$. Therefore by the Arzela-Ascoli theorem [48] $\overline{\mathcal{N} \mathbb{P}_{\lambda}}$ is compact . Clearly, $\mathbb{P}_{\lambda}$ is nonempty, bounded, convex, and closed subset of $C(\Delta)$. Hypotheses (ii) and (iv) lead to $\mathcal{N}: \mathbb{P}_{\lambda} \rightarrow C(\Delta)$ is a continuous operator in $\omega$.

Since all assumptions of Theorem 2.7 hold, then $\mathcal{N}$ has a fixed point in $\mathbb{P}_{\lambda}$.

\subsection{Special Cases}

In this part, we give some special cases that are also investigated according to our previous results.

Corollary 3.2. Under the hypotheses of Theorem 3.1 with $\varphi(t)=\phi(t)=t$, then the FQIE

$$
\omega(t)=h(t)+g(t, \omega(t)) \int_{0}^{t} \frac{[\psi(t)-\psi(\rho)]^{\alpha-1}}{\Gamma(\alpha)} \psi^{\prime}(\rho) f(\rho, \omega(\rho)) d \rho,
$$

has at least one solution $\omega \in \mathrm{C}(\Delta)$. 
Corollary 3.3. Under the hypotheses of Theorem 3.1 with $g(t, \omega(\varphi(t))) \equiv 1$, then the FFIE

$$
\omega(t)=h(t)+\int_{0}^{t} \frac{[\psi(t)-\psi(\rho)]^{\alpha-1}}{\Gamma(\alpha)} \psi^{\prime}(\rho) f(\rho, \omega(\phi(\rho))) d \rho,
$$

has at least one solution $\omega \in \mathrm{C}(\Delta)$.

Remark 3.4. If $\mathrm{b}=0$ and $\psi(\mathrm{t})=\phi(\mathrm{t})=\mathrm{t}$ in Corollary 3.3, we get the same result proven in [40].

Now putting $\alpha, \beta \rightarrow 1$, we get the following corollary:

Corollary 3.5. Under the hypotheses of Theorem 3.1 with $g(t, \omega(\varphi(t))) \equiv 1, h(t)=\omega_{0}$ and $\alpha=\beta=1$, then the following IE

$$
\omega(t)=\omega_{0}+\int_{0}^{t} \psi^{\prime}(\rho) f(\rho, \omega(\phi(\rho))) d \rho
$$

has at least one solution $\omega \in \mathrm{C}(\Delta)$ which is equivalent to the problem

$$
\omega_{\psi}^{\prime}(t)=f\left(t, \omega(\phi(t)), \omega(0)=\omega_{0},\right.
$$

where $\omega_{\psi}^{\prime}(t)=\frac{\omega^{\prime}(t)}{\psi^{\prime}(t)}$.

Remark 3.6. In Corollary 3.5, if $\psi(t)=\phi(t)=t$, and $b=0$ in the condition (v), then the following IE

$$
\left.\omega(t)=\omega_{0}+\int_{0}^{t} f(\rho, \omega(\rho))\right) d \rho
$$

has at least one solution $\omega \in \mathrm{C}(\Delta)$ is equivalent to the problem

$$
\omega^{\prime}(t)=f\left(t, \omega(\phi(t)), \omega(0)=\omega_{0},\right.
$$

which was proved in [48].

\section{Applications}

In this part, we apply the results of Section 3 to some FDEs. In view of these applications, we consider nonlinear Caputo FFDE, Reimann-Liouville FFDE and classical DE of the forms:

$$
\begin{aligned}
& \left\{\begin{array}{c}
R L \mathcal{D}_{0^{+}}^{\alpha, \psi} \omega(t)=f(t, \omega(\phi(t)), t \in \Delta=[0,1], \alpha \in(0,1), \\
J_{0^{+}}^{1-\alpha, \psi} \omega(0)=\omega_{0},
\end{array}\right. \\
& \left\{\begin{aligned}
\mathrm{C}_{\mathcal{D}_{0^{+}}^{\alpha, \psi} \omega(\mathrm{t})} & =\mathrm{f}(\mathrm{t}, \omega(\phi(t)), \mathrm{t} \in \Delta=[0,1], \alpha \in(0,1), \\
\omega(0) & =\omega_{0},
\end{aligned}\right.
\end{aligned}
$$

and

$$
\left\{\begin{array}{c}
\omega^{\prime}(t)=f(t, \omega(\phi(t)), t \in \Delta=[0,1] \\
\omega(0)=\omega_{0}
\end{array}\right.
$$

where ${ }^{R} \mathcal{D}_{0^{+}}^{\alpha, \psi}$ and ${ }^{C} \mathcal{D}_{0^{+}}^{\alpha, \psi}$ are the fractional derivatives in the sense of $R L$ and Caputo of order $\alpha$, respectively. 
Theorem 4.1. Under the hypotheses of Theorem 3.1 with

$$
\mathrm{h}(\mathrm{t}) \equiv \frac{\omega_{0}}{\Gamma(\alpha)}[\psi(\mathrm{t})-\psi(0)]^{\alpha-1} \text { and } \mathrm{g}(\mathrm{t}, \omega(\varphi(\mathrm{t}))) \equiv 1,
$$

then the $R L$ problem (4.1) has at least one solution $\omega \in \mathrm{C}(\Delta)$.

Proof. Operating with $\mathcal{J}_{0^{+}}^{\alpha, \psi}$ on $(4.1)$, we obtain $([4,21])$

$$
\omega(t)=c_{1}[\psi(t)-\psi(0)]^{\alpha-1}+\mathcal{J}_{0^{+}}^{\alpha, \psi} f(t, \omega(\phi(t)),
$$

for $c_{1} \in \mathbb{R}$. By means of the condition $\mathcal{J}_{0^{+}}^{1-\alpha, \psi} \omega(0)=\omega_{0}$, we get $c_{1}=\frac{\omega_{0}}{\Gamma(\alpha)}$. Hence

$$
\omega(t)=\frac{\omega_{0}}{\Gamma(\alpha)}[\psi(t)-\psi(0)]^{\alpha-1}+\int_{0}^{t} \frac{[\psi(t)-\psi(\rho)]^{\alpha-1}}{\Gamma(\alpha)} \psi^{\prime}(\rho) f(\rho, \omega(\phi(\rho))) d \rho, \quad t \in \Delta .
$$

Operating with ${ }^{\mathrm{RL}} \mathcal{D}_{0^{+}}^{\alpha, \psi}$ on FFIE (4.4) we obtain the RL problem (4.1). Moreover, the condition $J_{0^{+}}^{1-\alpha, \psi} \omega(0)=\omega_{0}$ is satisfied. Consequently, the equivalence between the problem (4.1) and the FFIE (4.4) is proved and then the results follow from Theorem 3.1 .

Theorem 4.2. Under the hypotheses of Theorem 3.1 with $\mathrm{h}(\mathrm{t}) \equiv \omega_{0}$ and $\mathrm{g}(\mathrm{t}, \omega(\varphi(\mathrm{t}))) \equiv 1$, then the Caputo problem (4.2) has at least one solution $\omega \in \mathrm{C}(\Delta)$.

Proof. Operating with $\mathcal{J}_{0^{+}}^{\alpha, \psi}$ on (4.2) we obtain we obtain ([21])

$$
\omega(t)=c_{0}+\mathcal{J}_{0^{+}}^{\alpha, \psi} f(t, \omega(\phi(t)),
$$

for $c_{0} \in \mathbb{R}$. By means of the condition $\omega(0)=\omega_{0}$, we get $c_{1}=\omega_{0}$. Hence

$$
\omega(t)=\omega_{0}+\int_{0}^{t} \frac{[\psi(t)-\psi(\rho)]^{\alpha-1}}{\Gamma(\alpha)} \psi^{\prime}(\rho) f(\rho, \omega(\phi(\rho))) d \rho, \quad t \in \Delta
$$

which by Theorem 3.1 has the desired solution.

Operating with ${ }^{C} \mathcal{D}_{0^{+}}^{\alpha, \psi}$ on (4.5) we obtain the problem (4.2). Therefore the equivalence between the problem (4.2) and the FFIE (4.5) is proved and then the results follow from Theorem 3.1.

In particular, if $\alpha=1$ and $\psi(t)=t$, then the problem (4.2) reduces to the problem (4.3). So, the IE

$$
\omega(t)=\omega_{0}+\int_{0}^{t} f(\rho, \omega(\phi(\rho))) d \rho, \quad t \in \Delta
$$

is equivalent to the problem (4.3). 


\section{Maximal and Minimal Solutions}

Definition 5.1. [50] The function $\mathfrak{Z}(t)$ is called a maximal solution of (1.1) if every solution of (1.1) on $\Delta$ satisfies

$$
\omega(t) \leqslant \mathfrak{Z}(t), \quad t \in \Delta .
$$

Whereas, the function $\mathfrak{N}(\mathrm{t})$ is called a minimal solution of (1.1) if every solution of (1.1) on $\Delta$ satisfies

$$
\omega(t) \geqslant \mathfrak{N}(t), \quad t \in \Delta
$$

To prove the existence of maximum and minimum solutions for (1.1), we need the following lemma:

Lemma 5.2. Assume that $\mathrm{f}(\mathrm{t}, \mathrm{\omega})$ is nondecreasing function in $\omega$. Let $\mathrm{g}(\mathrm{t}, \mathrm{\omega}), \mathrm{f}(\mathrm{t}, \mathrm{\omega})$ satisfy the hypotheses in Theorem 3.1 and let $\omega(t), \varpi(t)$ be continuous functions on $\Delta$ satisfying

$$
\begin{aligned}
& \omega(t) \leqslant h(t)+g\left(t, \omega(\varphi(t)) \mathcal{J}_{0^{+}}^{\alpha, \psi} f(t, \omega(\phi(t)),\right. \\
& \varpi(t) \geqslant h(t)+g\left(t, \varpi(\varphi(t)) \mathcal{J}_{0^{+}}^{\alpha, \psi} f(t, \varpi(\phi(t)),\right.
\end{aligned}
$$

where either (5.1) or (5.2) is strict. Then

$$
\omega(t)<\omega(t) .
$$

Proof. Assume that the conclusion (5.3) be false. Then there exists $t_{1}$ such that

$$
\omega\left(t_{1}\right)=\varpi\left(t_{1}\right), \quad t_{1}>0,
$$

and

$$
\omega(t)<\varpi(t), \quad 0<t<t_{1} .
$$

Since $f, g$ are monotone functions in $\omega$, it follows that

$$
\begin{aligned}
\omega\left(t_{1}\right) & \leqslant h\left(t_{1}\right)+g\left(t_{1}, \omega\left(\varphi\left(t_{1}\right)\right) J_{0^{+}}^{\alpha, \psi} f\left(t_{1}, \omega\left(\phi\left(t_{1}\right)\right)\right.\right. \\
& =h\left(t_{1}\right)+g\left(t_{1}, \omega\left(\varphi\left(t_{1}\right)\right) \int_{0}^{t_{1}} \frac{\left[\psi\left(t_{1}\right)-\psi(\rho)\right]^{\alpha-1}}{\Gamma(\alpha)} \psi^{\prime}(\rho) f(\rho, \omega(\phi(\rho))) d \rho\right. \\
& <h\left(t_{1}\right)+g\left(t_{1}, \varpi\left(\varphi\left(t_{1}\right)\right) \int_{0}^{t_{1}} \frac{\left[\psi\left(t_{1}\right)-\psi(\rho)\right]^{\alpha-1}}{\Gamma(\alpha)} \psi^{\prime}(\rho) f(\rho, \varpi(\phi(\rho))) d \rho\right. \\
& <\varpi\left(t_{1}\right) .
\end{aligned}
$$

This contradicts with our assumption (5.4). Hence

$$
\omega(t)<\varpi(t)
$$

Theorem 5.3. Suppose the hypotheses of Theorem 3.1 be fulfilled. In addition, if $f(t, \omega)$ is nondecreasing functions in $\omega$, then there exist maximal and minimal solutions of (1.1). 
Proof. Initially, we will prove the existence of maximal solution of (1.1). Let $\varepsilon>0$ be given. Now consider the following FQFIE

$$
\omega_{\mathcal{\varepsilon}}(t)=h(t)+g_{\varepsilon}\left(t, \omega_{\varepsilon}(\varphi(t)) J_{0^{+}}^{\alpha, \psi} f_{\varepsilon}\left(t, \omega_{\varepsilon}(\phi(t)),\right.\right.
$$

where

$$
f_{\mathcal{E}}\left(t, \omega_{\mathcal{\varepsilon}}(\phi(t))=f\left(t, \omega_{\mathcal{\varepsilon}}(\phi(t))+\varepsilon,\right.\right.
$$

and

$$
g_{\varepsilon}\left(t, \omega_{\varepsilon}(\varphi(t))=g\left(t, \omega_{\varepsilon}(\varphi(t))+\varepsilon .\right.\right.
$$

Obviously the functions $f_{\varepsilon}\left(t, \omega_{\varepsilon}\right)$ and $g_{\varepsilon}\left(t, \omega_{\varepsilon}\right)$ satisfy hypotheses (ii), (iv) and

$$
\begin{gathered}
\left|g_{\varepsilon}\left(t, \omega_{\varepsilon}\right)\right| \leqslant \eta+\varepsilon:=\eta^{\prime}, \\
\left|f_{\varepsilon}\left(t, \omega_{\varepsilon}\right)\right| \leqslant \eta(t)+\varepsilon+b|\omega|:=\eta^{\prime}(t)+b|\omega| .
\end{gathered}
$$

Consequently, the equation (5.5) has a continuous solution $\omega(t)$ due to Theorem 3.1.

Next, let $\varepsilon_{1}$ and $\varepsilon_{2}$ be such that $0<\varepsilon_{2}<\varepsilon_{1}<\varepsilon$. Then

$$
\begin{aligned}
\omega_{\varepsilon_{1}}(t) & =h(t)+g_{\varepsilon_{1}}\left(t, \omega_{\varepsilon_{1}}(\varphi(t)) J_{0^{+}}^{\alpha, \psi} f_{\varepsilon_{1}}\left(t, \omega_{\varepsilon_{1}}(\phi(t))\right.\right. \\
& =h(t)+\left(g ( t , \omega _ { \varepsilon _ { 1 } } ( \varphi ( t ) ) + \varepsilon _ { 1 } ) J _ { 0 ^ { + } } ^ { \alpha , \psi } \left(f\left(t, \omega_{\varepsilon_{1}}(\phi(t))+\varepsilon_{1}\right)\right.\right. \\
& >h(t)+\left(g ( t , \omega _ { \varepsilon _ { 1 } } ( \varphi ( t ) ) + \varepsilon _ { 2 } ) J _ { 0 ^ { + } } ^ { \alpha , \psi } \left(f\left(t, \omega_{\varepsilon_{1}}(\phi(t))+\varepsilon_{2}\right) .\right.\right.
\end{aligned}
$$

Also, we have

$$
\omega_{\varepsilon_{2}}(t)=h(t)+\left(g ( t , \omega _ { \varepsilon _ { 2 } } ( \varphi ( t ) ) + \varepsilon _ { 2 } ) \mathcal { J } _ { 0 ^ { + } } ^ { \alpha , \psi } \left(f\left(t, \omega_{\varepsilon_{2}}(\phi(t))+\varepsilon_{2}\right) .\right.\right.
$$

Applying Lemma 5.2, it follows from (5.6) and (5.7) that

$$
\omega_{\varepsilon_{2}}(t)<\omega_{\varepsilon_{1}}(t), \quad t \in \Delta .
$$

Let the operator $\mathcal{N}_{\varepsilon}: C(\Delta) \rightarrow C(\Delta)$ defined by

$$
\mathcal{N}_{\varepsilon} \omega_{\varepsilon}(t)=h(t)+g_{\varepsilon}\left(t, \omega_{\varepsilon}(\varphi(t)) J_{0^{+}}^{\alpha, \psi} f_{\varepsilon}\left(t, \omega_{\varepsilon}(\phi(t)) .\right.\right.
$$

By same reported arguments in proof of Theorem 3.1, the operator $\mathcal{N}_{\varepsilon}$ is uniformly bounded and equicontinuous. Hence, the Arzela-Ascoli Theorem shows that there exists a decreasing sequence $\varepsilon_{n}$ such that $\varepsilon_{n} \rightarrow 0$ as $n \rightarrow \infty$ and the uniform limit $\lim _{n \rightarrow \infty} \omega_{\varepsilon_{\mathfrak{n}}}(t)=\mathfrak{Z}(t)$ exists on $\Delta$. Since the functions $f_{\varepsilon_{\mathfrak{n}}}$ and $g_{\varepsilon_{n}}$ are continuous in the second argument, it follows that

$$
\mathfrak{Z}(t)=\lim _{n \rightarrow \infty} \omega_{\mathcal{E}_{n}}(t)=h(t)+g\left(t, \mathfrak{Z}(\varphi(t)) \mathcal{J}_{0^{+}}^{\alpha, \psi} f(t, \mathfrak{Z}(\phi(t)) .\right.
$$

This proves that $\mathfrak{Z}(t)$ is a solution of (1.1).

Finally, we will show that $\mathfrak{Z}(t)$ is maximal solution of (1.1). Indeed, let $\omega(t)$ be any solution of (1.1). Then we have

$$
\begin{aligned}
\omega_{\mathcal{E}}(t) & =h(t)+g_{\mathcal{E}}\left(t, \omega_{\mathcal{\varepsilon}}(\varphi(t)) \mathcal{J}_{0^{+}}^{\alpha, \psi} f_{\mathcal{E}}\left(t, \omega_{\mathcal{\varepsilon}}(\phi(t))\right.\right. \\
& >h(t)+g\left(t, \omega_{\mathcal{E}}(\varphi(t)) \mathcal{J}_{0^{+}}^{\alpha, \psi} f\left(t, \omega_{\mathcal{\varepsilon}}(\phi(t)),\right.\right.
\end{aligned}
$$


and

$$
\omega(t)=h(t)+g\left(t, \omega(\varphi(t)) \mathcal{J}_{0^{+}}^{\alpha, \psi} f(t, \omega(\phi(t)),\right.
$$

Applying Lemma 5.2, we get

$$
\omega_{\varepsilon}(t)>\omega(t), \quad \text { for all } t \in \Delta .
$$

from the uniqueness of the maximal solution (see [50]), it is obvious that the uniform limit

$$
\lim _{\varepsilon \rightarrow 0} \omega_{\varepsilon}(t)=\mathfrak{Z}(t)
$$

exists on $\Delta$.

Likewise, we can demonstrate that there exists a minimal solution of (1.1).

\section{6. conclusion}

In this article, we have considered the generalized fractional quadratic functional integral equations involving $\psi$-fractional operators. Some existence results for at least one continuous solution for the proposed equations have investigated. Fractional differential equations with respect to another function $\psi$ were given as an application. We have applied the Schauder fixed point technique and nonlinear functional analysis to discuss these results. Further, we have proven the existence of maximal and minimal solutions for these equations. Many results of equations that contain classical fractional operators are obtained as special cases of (1.1). The reported results in this paper are recent and significantly contribute to the existing literature on the subject. For future work, the problems studied in this paper can be extended to cover other kinds of fractional quadratic functional integral equations involving $\psi$-Hilfer fractional operator.

\section{Acknowledgment}

The author are grateful to the referees for the careful reading of the paper and for their remarks.

\section{References}

[1] Diethelm K (2010). "The analysis of fractional differential equations". Lecture Notes in Mathematics, Springer-verlag Berlin Heidelberg.

[2] Hilfer R (2000). "Applications of Fractional Calculus in Physics". World Scientific, Singapore.

[3] Lakshmikantham V and Leela S (2009). "Theory of fractional dynamic systems". Cambridge Scientific Publisher LTD. Cambridge.

[4] Kilbas AA, Srivastava HM and Trujillo JJ (2006). "Theory and Applications of Fractional Differential Equations". North-Holland Math Stud, Elsevier, Amsterdam, 204.

[5] Miller KS and Ross B (1993). "An introduction to the fractional calculus and fractional differential equations". New York: John Wiley \& Sons, Inc.

[6] Podlubny I (1999). "Fractional differential equations". San Diego, CA: Academic Press.

[7] Sabatier J, Agrawal OP and Machado JAT (2007). "Advances in fractional calculus". Springer, Dordrecht.

[8] Jarad F, Abdeljawad $T$ and Hammouch Z (2018). On a class of ordinary differential equations in the frame of Atangana-Baleanu fractional derivative. Chaos Solitons Fractals 117:16-20. https://doi.org/10.1016/j.chaos.2018.10.006 
[9] Abdeljawad T (2017). Fractional operators with exponential kernels and a Lyapunov type inequality. Adv. Differ. Equ. 2017(1):313. https://doi.org/10.1186/s13662-017-1285-0

[10] Abdeljawad T (2011). On Riemann and Caputo fractional differences. Comput. Math. Appl. 62(3):16021611. https://doi.org/10.1016/j.camwa.2011.03.036

[11] Kilbas AA, Rivero M and Trujillo JJ (2003). Existence and uniqueness theorems for differential equations of fractional order in weighted spaces of continuous functions. Frac. Calc. Appl. Anal. 6 (4): 363-400.

[12] Baleanu D and Trujillo JJ (2008). On exact solutions of a class of fractional Euler-Lagrange equations. Nonlinear Dyn. 52(4): 331-335. https://doi.org/10.1007/s11071-007-9281-7

[13] Caputo M (1967). Linear model of dissipation whose Q is almost frequency independent. II. Geophysical J. International 13(5): 529-539. https://doi.org/10.1111/j.1365-246X.1967.tb02303.x

[14] Hadamard J (1892). Essai sur l' 'etude des fonctions donn'ees par leur d'eveloppement de Taylor. Journal de Math'ematiques Pures et Appliqu'ees 4(8): 101-186.

[15] Katugampola UN (2014). A new approach to generalized fractional derivatives. Bull. Math. Anal. Appl. 6(4): 1-15.

[16] Prabhakar TR (1971). A singular integral equation with a generalized Mittag-Leffler function in the kernel. Yokohama. Math. J. 19: 7-15.

[17] Jarad F, Abdeljawad T and Baleanu D (2012). Caputo-type modification of the Hadamard fractional derivatives. Adv. Differ. Equ. 2012 (142): 8. https://doi.org/10.1186/1687-1847-2012-142

[18] Almeida R (2017). A Gronwall inequality for a general Caputo fractional operator. arXiv preprint arXiv:1705.10079. https://arxiv.org/abs/1705.10079

[19] Caputo M and Fabrizio M (2015). A new Definition of Fractional Derivative without Singular Kernel. Progr. Fract. Differ. Appl. 1(2): 73-85. https://doi.org/10.12785/pfda/010201

[20] Atangana A and Baleanu D (2016). New fractional derivatives with nonlocal and nonsingular kernel: theory and application to heat transfer model. Therm. Sci. 20(2): 763-69. https://doi.org/10.2298/TSCI160111018A

[21] Almeida R (2017). A Caputo fractional derivative of a function with respect to another function. Commun. Nonlinear Sci. Numer. Simul. 44: 460-481. https://doi.org/10.1016/j.cnsns.2016.09.006

[22] Sousa JVC and Oliveira EC (2018). On the $\psi$-Hilfer fractional derivative. Commun. Nonlinear Sci. Numer. Simul. 60: 72-91. https://doi.org/10.1016/j.cnsns.2018.01.005

[23] Jarad F, Ug̃urlu E, Abdeljawad T and Baleanu D (2017). On a new class of fractional operators. Adv. Differ. Equ. 2017(1): 247. https://doi.org/10.1186/s13662-017-1306-z

[24] Jarad F, Abdeljawad T and Baleanu D (2017). On the generalized fractional derivatives and their Caputo modification. J. Nonlinear Sci. Appl. 10(5): 2607-2619.

[25] Abdeljawad T (2015). On conformable fractional calculus. J. Comput. Appl. Math. 279: 57-66. https://doi.org/10.1016/j.cam.2014.10.016

[26] Argyros IK (1985). Quadratic equations and applications to Chandrasekhar's and related equations. Bull. Aust. Math. Soc. 32: 275-292. https://doi.org/10.1017/S0004972700009953

[27] Argyros IK (1992). On a class of quadratic integral equations with perturbation. Funct. Approximatio Comment. Math. 20: 51-63.

[28] Bana's J, Caballero J, Rocha J and Sadarangani K (2005). Monotonic solutions of a class of quadratic integral equations of Volterra type. Comput. Math. Appl. 49(5-6): 943-952. https://doi.org/10.1016/j.camwa.2003.11.001

[29] Bana's J, Martin JR and Sadarangani K (2006). On solutions of a quadratic integral equation of Hammerstein type. Math. Comput. Modelling 43(1-2): 97-104. https://doi.org/10.1016/j.mcm.2005.04.017

[30] Bana's J and Martinon A (2004). Monotonic solutions of a quadratic integral equation of Volterra type. Comput. Math. Appl. 47(2-3): 271-279, 2004. https://doi.org/10.1016/S0898-1221(04)90024-7

[31] Bana's J, Lecko M and El-Sayed WG (1998). Existence theorems for some quadratic integral equations. J. Math. Anal. Appl. 222(1): 276-285. https://doi.org/10.1006/jmaa.1998.5941

[32] Darwish MA (2008). On monotonic solutions of a singular quadratic integral equation with supremum. Dyn. Syst. Appl. 17(3-4): 539-549.

[33] El Borai MM, El-Sayed WG and Abbas MI (2007). Monotonic solutions of a class of quadratic singular integral equations of Volterra type. Int. J. Contemp. Math. Sci. 2(1-4): 89-102.

[34] Bana's J and Rzepka B (2007). Monotonic solutions of a quadratic integral equation of fractional order. J. Math. Anal. Appl. 332(2): 1371-1379. https://doi.org/10.1016/j.jmaa.2006.11.008

[35] El-Sayed AMA and Hashem HHG (2008). Caratheodory type theorem for a nonlinear quadratic integral equation. Math. Sci. Res. J. 12(4): 88-95. 
[36] El-Sayed AMA and Hashem HHG (2008). Integrable and continuous solutions of a nonlinear quadratic integral equation. Electron. J. Qual. Theory Differ. Equ. 2008(25): 1-10. https://doi.org/10.14232/ejqtde.2008.1.25

[37] El-Sayed AMA and Hashem HHG (2008). Integrable solutions for quadratic Hammerstein and quadratic Urysohn functional integral equations. Commentat. Math. 48(2): 199-207. https://doi.org/10.14708/cm.v48i2.5272

[38] El-Sayed AMA and Hashem HHG (2009). Monotonic solutions of functional integral and differential equations of fractional order. Electron. J. Qual. Theory Differ. Equ. 2009: 1-8. https://doi.org/10.14708/cm.v48i2.5272

[39] El-Sayed AMA and Hashem HHG (2010). Monotonic positive solution of a nonlinear quadratic functional integral equation. Appl. Math. Comput. 216(9): 2576-2580. https://doi.org/10.1016/j.amc.2010.03.099

[40] El-Sayed AMA, Gaafar FM and Hashem HHG (2004). On the maximal and minimal solutions of arbitraryorders nonlinear functional integral and differential equations. Math. Sci. Res. J. 8(11): 336-348.

[41] Abdo MS, Ibrahim AG and Panchal SK (2019). Nonlinear implicit fractional differential equation involving $\psi$-Caputo fractional derivative. Proc. Jangjeon Math. Soc. 22(3): 387-400. http://dx.doi.org/10.17777/pjms2019.22.3.387

[42] Abdo MS, Shah K, Panchal SK and Wahash HA (2020) Existence and Ulam stability results of a coupled system for terminal value problems involving $\psi$-Hilfer fractional operator. Adv. Differ. Equ. 2020: 316. https://doi.org/10.1186/s13662-020-02775-x

[43] Abdo MS, Panchal SK and Saeed AM (2019). Fractional boundary value problem with $\psi$-Caputo fractional derivative. Proc. Indian Acad. Sci. Math. Sci. 129(5): 65. https://doi.org/10.1007/s12044-019-0514-8

[44] Wahash HA, Abdo MS, Saeed AM and Panchal SK (2020). Singular fractional differential equations with భ-Caputo operator and modified Picard's iterative method. Appl. Math. E-Notes 20: 215-229.

[45] Sousa JVC, de Oliveira EC and Kucche KD (2019). On the Fractional Functional Differential Equation with Abstract Volterra Operator. Bull. Braz. Math. Soc. New Series 50: 803-822. https://doi.org/10.1007/s00574-019-00139-y

[46] El-Sayed AMA and Hashem HHG (2013). Existence results for nonlinear quadratic functional integral equations of fractional orders. Miskolc Math. Notes 14(1): 79-88. https://doi.org/10.18514/MMN.2013.578

[47] Salem HAH (2011). On the quadratic integral equations and their applications. Comput. Math. Appl. 62(8): 2931-2943. https://doi.org/10.1016/j.camwa.2011.07.070

[48] Curtain RF and Pritchard AJ (1977). "Functional analysis in modern applied mathematics". Ser. Mathematics in Science and Engineering. London, New York: Academic Press, 132.

[49] Granas A and Dugundji J (2003). "Fixed Point Theory". Springer-Verlag: New York, NY, USA.

[50] Lakshmikantham V and Leela S (1969). "Differential and integral inequalities: Theory and applications". Vol. I: Ordinary differential equations, ser. Mathematics in Science and Engineering. New York-London: Academic Press, 55. 